\title{
DEMOGRAPHIC CHANGES IN THE POPULATION OF BULGARIA AS CHALLENGES FOR ANAESTHESIOLOGISTS
}

\author{
Vladimir R. Radev, \\ Radko N. Radev, \\ Daniela A. Arabadzhieva ${ }^{1}$, \\ Vladin I. Petrov', \\ Nikolay H. Kolev ${ }^{2}$
}

\author{
Department of Anaesthesiology and \\ Intensive Care, \\ Medical Faculty, \\ Medical University - Pleven, \\ Bulgaria \\ ${ }^{1}$ Department of Anaesthesiology and \\ Intensive Care, \\ University Hospital - Rouse, \\ Bulgaria \\ ${ }^{2}$ Department of Urology, \\ University Hospital - Pleven, \\ Bulgaria
}

\section{Summary}

In the past two decades, the demographic crisis in Bulgaria has been widely debated. According to the National Statistical Institute (NSI), the proportion of people aged 65 and over is increasing, despite the general population decline in the country. To investigate whether the age structure of patients operated on has changed during the past 10 years and assess the need to reorganize the training of anesthetists, a retrospective study in the anesthesia electronic database of the University Hospital in Rouse was carried out. The data of anesthesia performed by anesthesiologists in 2005, 2010 and 2015 in patients aged 65 and over were summarized. The distribution of these patients by ASA Physical Classification System and frequency of complications during anesthesia were analyzed. The average annual number of anaesthesias for the three years was almost unchanged and amounted to about 5500 . The proportion of patients aged 65 and over increased from $30.54 \%$ in 2005 to $35.72 \%$ in 2010 , and $37.81 \%$ in 2015 . The distribution of patients in according to the ASA Classification changed significantly: the percentage of patients ASA I-II dropped from $36.19 \%$ in 2005 to $11.85 \%$ in 2015. The patients in the ASA III-IV groups for the three analyzed years was $41.86 \%, 58.66 \%$ and $65.30 \%$, respectively, and this was mainly due to the increase in the ASA IV patients. It is necessary to build multidisciplinary teams and prepare specialized protocols for behavior in geriatric patients. It is imperative that anesthesiologists possess specialized knowledge about age-related organ changes and their effects on anesthesia.

Key words: Geriatric anesthesiology, Bulgarian demographics, descriptive statistic

\section{Introduction}

The proportion of adults in Bulgaria increases and this increase leads to significant changes in population structure. In older patients, adverse effects and complications associated with anesthesia and surgery are more likely to occur for three main reasons: first, they have at least one but usually more than one co-morbidities, second, they have changed and impaired pharmacokinetics and pharmacodynamics, and third - they have reduced organ function [1].

The aim of this study was to identify the changes
of the age structure of patients operated on in a

The aim of this study was to identify the changes
of the age structure of patients operated on in a
Received: November 23, 2016

Revision received: December 13, 2016

Accepted: December 20, 2016 
regional multi-profile hospital for 10 years and the need for reorganizing the education and training process of anaesthetists.

\section{Materials and Methods}

The electronic database of anaesthetic procedures performed in the University Hospital in Rouse was studied retrospectively. The database has been supported by anesthesiologists since 2003. At the end of 2016, it contained information about 70000 anesthetic procedures performed on patients from the pediatric, purulent-septic surgery, vascular surgery, orthopedics and traumatology, urology, plastic and reconstructive surgery and the ENT departments. There was no information about anesthesia give in the obstetrics and gynaecology departments. The database concerned anesthesia performed by anesthesiologists and no data on local anesthesia given by operating room teams. The information of patients aged 65 and older, their distribution by ASA Classification and the overall incidence of complications was derived using proper filters.

\section{Results}

The information about the number and structure of the population in Bulgaria according to NSI data is summarized in Table 1 and Figure 1.

The data in Table 1 clearly show the trend of increase in the number of people aged 65 and over, despite the reduced number of overall population. Figure 1 shows the higher increase rate of the elderly among those aged 75-79.
Table 2 summarizes the total number of anesthesias and the proportion of patients 65 and older during the three years analyzed.

The significantly smaller number of operations/anesthesias in 2015 than in 2010 $(\mathrm{p}=0.025)$ can be attributed to changes in regulations introduced by the National Framework Agreement in 2015 and limited numbers of admissions. Another major factor was the opening of a new general hospital that attracted part of the patients.

It can be seen from Figure 2 that the number of the patients in the group 70-79 decreased, while that of patients in the other three age groups increased. The most significant increase in the number of patients aged 80-89 in 2005, 2010 and 2015 was - $219(13.21 \%) 401(19.14 \%)$ and 465 (25.56\%), respectively.

Table 3 presents data on the distribution of patients according to ASA Classification. The figures clearly show that in both planned and emergency anesthesia the number of patients with clinically significant or life-threatening conditions had increased.

Table 4 presents data on the total number of complications registered in patients older than 60. Various types of complications are included, divided in to several groups: complications of respiratory system-oxygenation-gas exchange, the cardiovascular system, systemic reactions and systemic damage, injuries, lesions, blood, electrolyte balance, of the central and peripheral nervous system and neuromuscular transmission, technical defects, deficiencies, errors and specific complications associated with regional anesthesia.

Table 1. Prognosis of NSI for distribution of population by age, based on the results of the 2011 census

\begin{tabular}{lllll}
\hline Year & $\mathbf{2 0 1 1}$ & $\mathbf{2 0 1 5}$ & $\mathbf{2 0 2 0}$ & $\mathbf{2 0 2 5}$ \\
\hline Age & 7504868 & 7168009 & 6966607 & 6760045 \\
\hline $65-69$ & 400984 & 492853 & & \\
\hline $70-74$ & 337778 & 360949 & 453504 & 433337 \\
\hline $75-79$ & 297819 & 275871 & 435620 & 405419 \\
\hline $80-84$ & 188779 & 205693 & 295035 & 362799 \\
\hline $85-89$ & 85437 & 99039 & 194023 & 214671 \\
\hline $90-94$ & 17156 & 30892 & 111968 & 110327 \\
\hline $95-99$ & 3185 & 3886 & 37516 & 44566 \\
\hline $100+$ & 233 & 166 & 7277 & 9187 \\
\hline Total & & $1469369 / \mathbf{2 0 . 5 0 \%}$ & $1635226 / \mathbf{2 2 . 0 4 \%}$ & $1580747 / \mathbf{2 3 . 3 8 \%}$ \\
\hline
\end{tabular}




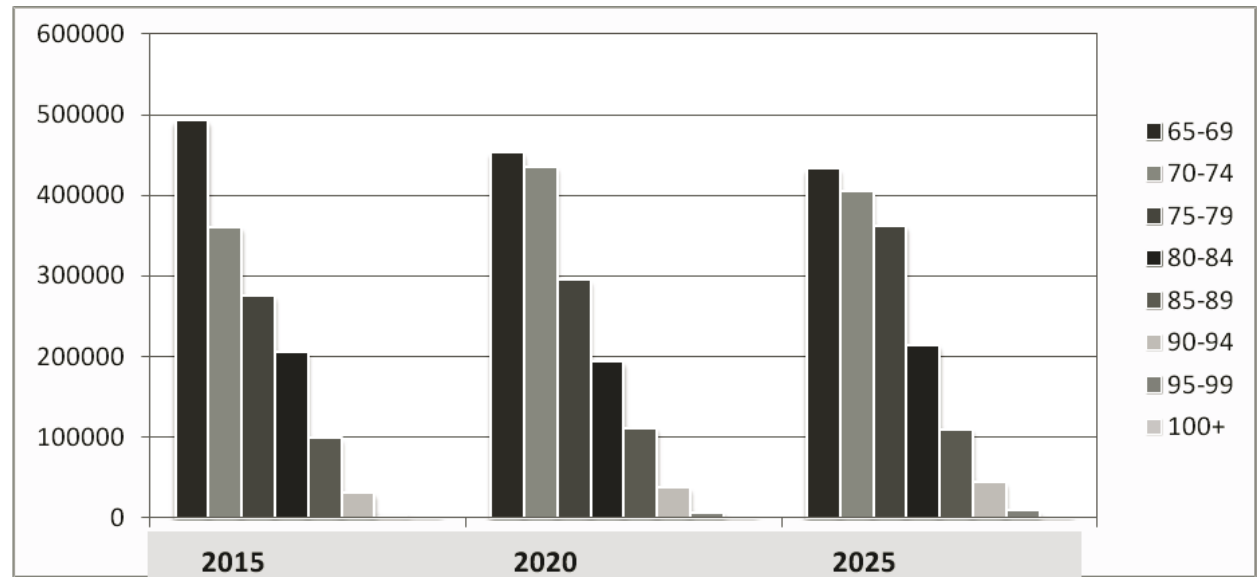

Figure 1. Distribution of people aged $65+$ by NSI and prognosis of changes

Table 2. Total number of anesthesia conducted and proportion of patients aged $\geq 65$

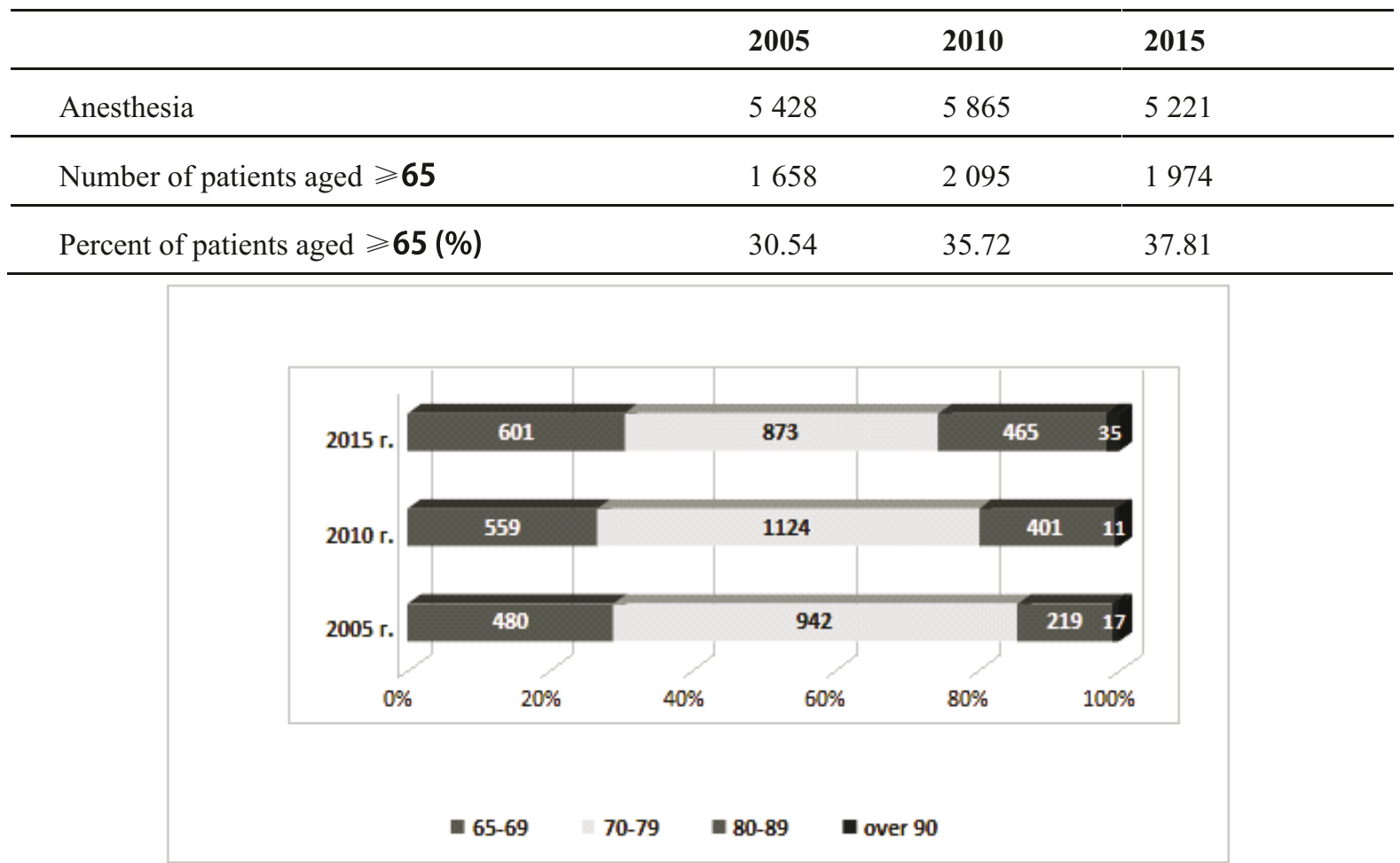

Figure 2. Distribution of patient subgroups by age (65-69, 70-79, 80-89, and 90+)

Table 3. Distribution of patients aged $\geqslant 65$ according to ASA Classification

\begin{tabular}{lllllll}
\hline & 2005 & \multicolumn{3}{l}{2010} & \multicolumn{2}{l}{2015} \\
\cline { 2 - 7 } & Number & $\%$ & Number & $\%$ & Number & $\%$ \\
\hline ASA I -II & 600 & 36.19 & 330 & 15.75 & 234 & 11.85 \\
\hline ASA III-IV & 694 & 41.86 & 1229 & 58.66 & 1289 & 65.30 \\
\hline ASA V & 1 & 0.06 & 2 & 0.10 & 5 & 0.25 \\
\hline ASA IE*- IIE & 116 & 7.00 & 89 & 4.25 & 53 & 2.69 \\
\hline ASA IIIE- IVE & 232 & 13.99 & 417 & 19.90 & 368 & 18.64 \\
\hline ASA VE & 15 & 0.90 & 28 & 1.34 & 25 & 1.27 \\
\hline *Emergency cases & & & & & \\
\hline
\end{tabular}


Table 4. Complications registered during anesthesia

\begin{tabular}{lll}
\hline & $\begin{array}{l}\text { Total registered } \\
\text { complications }\end{array}$ & $\begin{array}{l}\text { Complications in } \\
\text { patients } \geqslant \mathbf{6 5}\end{array}$ \\
\hline 2005 & 938 & 422 \\
\hline 2010 & 774 & 442 \\
\hline 2015 & 631 & 368 \\
\hline
\end{tabular}

Complication rates depend on many factors, including the accepted definitions [2], grades of severity - applied or not, mode of communication - anonymous or open, as well as what was reported. The department has adopted a European standard 5-grade scale to assess the severity of complications during anesthesia, in which 1 corresponds to no need for hospital stay to recover from anesthesia and additional monitoring, and $\mathrm{V}$ refers to lethal outcome. Complications were reported voluntarily and openly, regardless of degree of severity. Under such schemes for assessment and reporting, the normal frequency of complications, according to medical literature is $18-32 \%$. On the other hand, if only complications that are potentially of legal importance are reported and recorded, the rate drops to values below $0.5 \%$.

Table 4 shows that in 2010 and 2015 more than half of the complications registered occurred in patients aged $\geqslant 65$, and these patients were approximately one-third of the total number of patients. According to data from specialized publications, elderly patients have longer average hospital stay, higher costs for treatment, greater risk of developing complications in operations and higher hospital mortality [3].

\section{Discussion}

Over the last two decades, the demographic crises in Bulgaria became obvious. On the one hand, it can be attributed to ageing of the population and the increasing number of people over 65 years old. On the other hand, it is the result from the negative population growth and emigration. Although the number of patients over 65 years old in University Hospital Rouse decreased, their relative share increased from $30.54 \%$ in 2005 to $37.81 \%$ in 2015 . The number of patients with ASA III and ASA IV and of complications during anesthesia also went up.
The challenges for anesthesiologists are determined by many factors - altered mental status and the risk of developing cognitive impairment and delirium, limited function of almost all systems - cardiovascular, respiratory, excretory, problems with thermoregulation, metabolic function, electrolyte balance, problems with permanent medication, and problems caused by surgical disease for which they are hospitalized.

\section{Conclusions}

The elderly need care provided by multidisciplinary teams including surgeons, anesthesiologists, therapists, physiotherapists, nutritionists and other medical specialists with expertise in the field of geriatrics. Designing specialized protocols for preoperative evaluation and preparation of patients, intraoperative management, and postoperative care and rehabilitation would be of great benefit for both health care providers and patients.

\section{References}

1. Harwood TN. Optimizing outcome in the very elderly surgical patient. Curr Opin Anaesthesiol. 2000;13(3):327-32.

2. Bijker JB, van Klei WA, Kappen $\mathrm{TH}$, van Wolfswinkel L, Moons KG, Kalkman CJ. Incidence of intraoperative hypotension as a function of the chosen definition: literature definitions applied to a retrospective cohort using automated data collection. Anesthesiology. 2007;107(2):213-20.

3. Hall MJ, Levant S, DeFrances CJ. Trends in inpatient hospital deaths: National Hospital Discharge Survey, 2000-2010. NCHS Data Brief. 2013;(118):1-8. 